$\mathbb{P}$ Periodica Polytechnica

Electrical Engineering and Computer Science

58(2), pp. 69-79 (2014)

DOI:10.3311/PPee.7373

Creative Commons Attribution (i)

RESEARCH ARTICLE

\section{Inclined Span Modelling by a Given Levelled Span for OHL Design}

Alen Hatibovic

RECEIVED 21 February 2014; ACCePted After REVISION 4 April 2014

\section{Abstract}

An overhead line (OHL) design is more difficult in a hilly terrain than that is in a flat terrain. One of the reasons is presence of inclined spans where the vertical distance between the support points is not zero. The algorithms for calculations in inclined spans are available in professional literatures but these are more complicated than those concerned to levelled spans. Creating a link between the two span types, the recent paper shows a mathematical solution for modelling an inclined span by given data for a levelled one and a freely selected vertical distance between the support points. This distance defines the span inclination. The method developed is based on the fact that the conductor curves in each span between the two deadend towers are parts of the same catenary, independently of the span inclination. The main discussion regards to the catenary, but its parabolic approximation is also mentioned.

The derived new relations concerned to the conductor sag give a possibility to examine and discuss an existing approximate relation between the catenary sags in inclined and levelled spans, which can be found in earlier literatures. Having both the new and earlier relations, the error produced by the use of the approximate relation can be exactly obtained at an arbitrary point of the span.

\section{Keywords}

overhead lines $\cdot$ catenary $\cdot$ parabola $\cdot$ conductor curve $\cdot$ sag $\cdot$ levelled span $\cdot$ inclined span

\section{Introduction and background}

From the aspect of OHL design a levelled span is a simpler type of a span. As the support points are on the same elevation, the lowest point of the conductor is located at a mid-span [6] and thus the sag calculation is not difficult. The levelled spans are shown in Figure 1. The other type of a span is an inclined one [1,5] and is shown in Figure 2 [10]. Due to different heights of the support points, the lowest point of the conductor is moved from the mid-span and thus all calculations are more complicated [8].

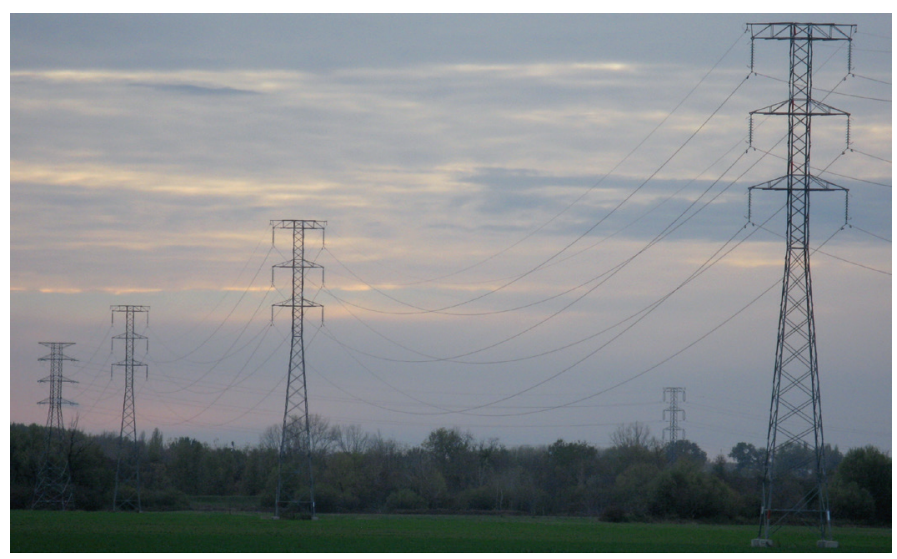

Fig. 1. OHL with levelled spans

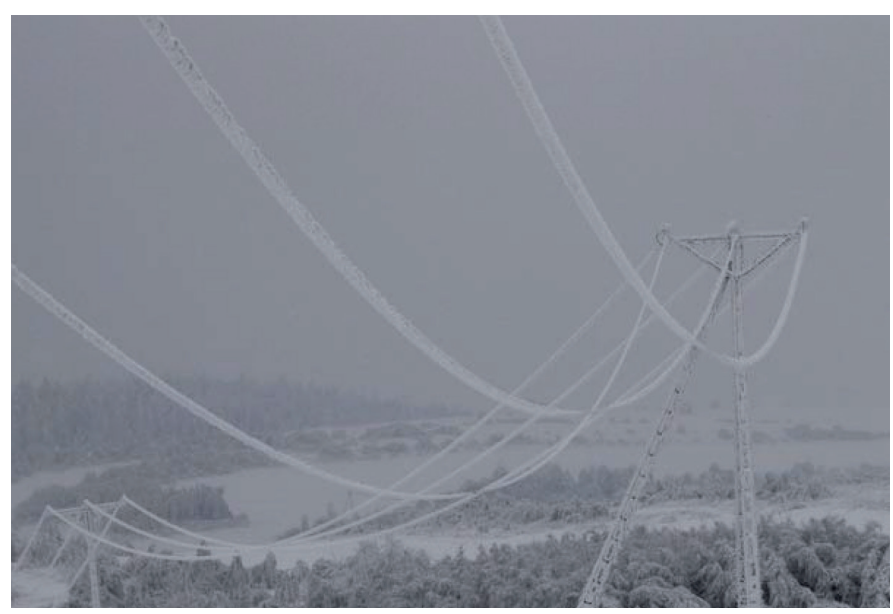

Fig. 2. OHL with inclined spans

\section{Alen Hatibovic}

Electrical Design Engineer, H-6500 Baja, Szivárvány u. 40, Hungary e-mail: hatibovic.alen@gmail.com
2014582 
The equations for the conductor curve and the sag in a levelled span can be obtained if the following input data are known: span length, $S$, catenary constant, $c$ and height of the support points, $h_{1}[2,3]$. Beside the freely chosen vertical distance $(\Delta h)$ between the support points [7], the listed data are sufficient for determining the equations for the conductor curve and the sag concerned to a formed inclined span. It is shown in the recent paper by using the new method named inclined span modelling by a given levelled span (or shortly inclined span modelling). A given levelled span here means that $S, c$ and $h_{1}$ data are given. While modelling, $S$ and $c$ have to remain unchanged. It can be reached for instance, in spans between dead end towers. The mentioned limitation is in connection with the following facts. In the case of the post line insulators and dead end (tension) insulators, the attachment points of the conductors cannot move in the direction of the line, therefore different catenary constants (different conductor tensions) do not change their position. On the other hand, suspension insulators can move freely in the direction of the line, therefore different catenary constants will change the length of the span. The movement of the suspension insulators may lead to substantial change in sags in the case of hilly terrain and long spans. There are several simplified methods for the calculation of this situation and the finite element analysis can be used as well.

Due to the specificity of the actual topic, the following abbreviations are used in the paper:

$y_{l e v}(x)$ - equation of the conductor curve in a levelled span,

$y_{\text {inc }}(x)$ - equation of the conductor curve in an inclined span,

$y_{\text {line lev }}(x)$ - equation of the straight line connecting the support points in a levelled span,

$y_{\text {line inc }}(x)$ - equation of the straight line connecting the support points in an inclined span,

$D_{\text {lev }}(x)$ - sag equation in a levelled span,

$D_{\text {inc }}(x)$ - sag equation in an inclined span,

$D_{\text {lev }}(S / 2)$ - mid-span sag in a levelled span,

$D_{\text {inc }}(S / 2)$ - mid-span sag in an inclined span,

$D_{\text {lev } \max }-$ maximum sag in a levelled span,

$D_{\text {inc } \max }$ - maximum sag in an inclined span,

$\Delta D(x)$ - equation of the difference between the sags in inclined and levelled spans,

$\Delta D(S / 2)$ - difference between the mid-span sags in inclined and levelled spans,

$\Delta D_{\text {max }}$ - difference between the maximum sags in inclined and levelled spans.

\section{Inclined span modelling and its use in practice 2.1 Method introduction}

In order to explain adequately the method for modelling an inclined span, four curves with common $S$ and $c$ for each one are drawn in Figure 3. The angle of the span inclination is marked as $\psi$.

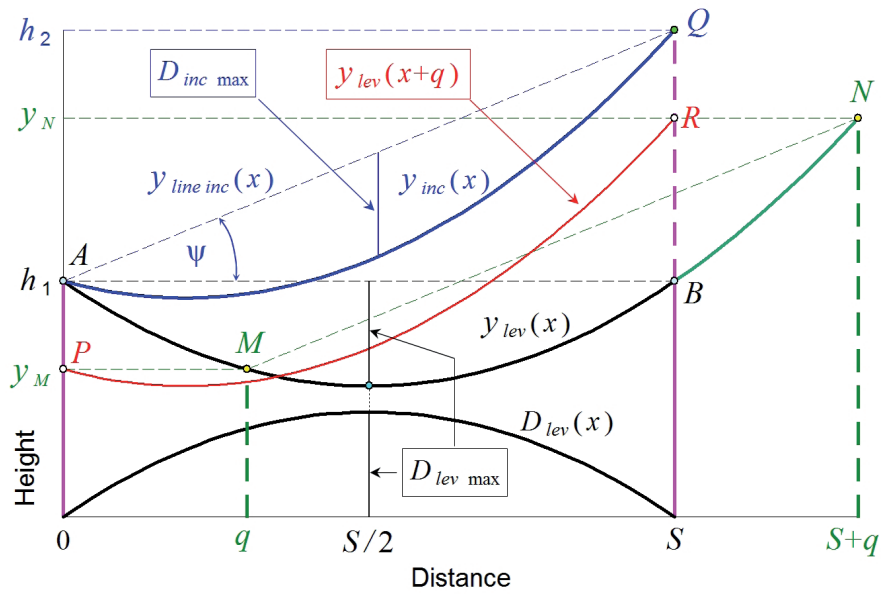

Fig. 3. Curves for explaining the inclined span modelling by a given levelled span

The initial curve is the one in a levelled span, drawn from the support point $A\left(0 ; h_{1}\right)$ to point $B\left(S ; h_{1}\right)$. The equation of this curve is given by (1) or (2) and is defined on the interval $[0, S]$.

$$
\begin{aligned}
& y_{\text {lev }}(x)=c \cdot \cosh \frac{x-S / 2}{c}-c \cdot \cosh \frac{S}{2 c}+h_{1} \\
& y_{\text {lev }}(x)=c \cdot \cosh \frac{x-S / 2}{c}-c+h_{1}-D_{\text {lev } \max }
\end{aligned}
$$

Using the previous equations, two versions of the sag equation (also called as sag formula) concerned to a levelled span can be obtained in the following way:

$$
\begin{gathered}
D_{\text {lev }}(x)=h_{1}-y_{\text {lev }}(x)=c \cdot \cosh \frac{S}{2 c}-c \cdot \cosh \frac{x-S / 2}{c} \\
D_{\text {lev }}(x)=D_{\text {lev } \max }-c \cdot \cosh \frac{x-S / 2}{c}+c
\end{gathered}
$$

Each of four preceding equations is a function of $x$, so it can be applied at any point of the span.

Extending the curve of $y_{\text {lev }}(x)$ on the interval $(S, S+q]$ and omitting its part on the interval $[0, q)$ we get the conductor curve in an inclined span drawn from point $M\left(q ; y_{M}\right)$ to point $N\left(S+q ; y_{N}\right)$, where $0<q<S, y_{M}=y_{l e v}(q)$ and $y_{N}=y_{l e v}(S+q)$. This way the created curve has the same equation as the initial one does, but it is defined on the interval $[q, S+q]$ :

$$
\begin{gathered}
(A B)_{\text {curve }}=y_{\text {lev }}(x) \quad x \in[0, S] \\
(M N)_{\text {curve }}=y_{\text {lev }}(x) \quad x \in[q, S+q]
\end{gathered}
$$

Note that both curves, $A B$ and $M N$, are the parts of the same catenary. Thus, the catenary constant is equal in two cases. Furthermore, the span length remained unchanged.

The following step is the displacement of the curve $M N$ so that point $M$ is set at point $A$. To reach that, the actual curve has to be translated horizontally $q$ units to the left, and vertically 
$h_{1}-y_{M}$ units upward. The horizontal translation produces the curve $P R$ and then the performed vertical translation gives the final curve $A Q$ :

$$
\begin{gathered}
(P R)_{\text {curve }}=y_{\text {lev }}(x+q) \quad x \in[0, S] \\
(A Q)_{\text {curve }}=y_{\text {lev }}(x+q)+h_{1}-y_{\text {lev }}(q) \quad x \in[0, S]
\end{gathered}
$$

Both translations are made by an appropriate use of the equation concerned to a levelled span, $y_{l e v}(x)$. The equation of the final curve drawn from point $A$ to point $Q$ is marked below as $y_{\text {inc }}(x)$, and is defined on the interval $[0, S]$. Considering (9) it is expressed by (10).

$$
\begin{gathered}
h_{1}-y_{\text {lev }}(q)=c \cdot \cosh \frac{S}{2 c}-c \cdot \cosh \frac{q-S / 2}{c} \\
y_{\text {inc }}(x)=c \cdot \cosh \frac{x-S / 2+q}{c}+h_{1}-c \cdot \cosh \frac{q-S / 2}{c}
\end{gathered}
$$

The rearrange of (10) yields (11). (The complete deduction is given in Appendix A.)

$$
y_{\text {inc }}(x)=2 c \cdot \sinh \frac{x}{2 c} \cdot \sinh \left(\frac{x-S}{2 c}+\frac{q}{c}\right)+h_{1}
$$

The next step is the determination of $q=q\left(S, c, h_{1}, h_{2}\right)$, i.e. expressing $q$ by $S, c, h_{1}$ and $h_{2}$. The quotient $q / c$ can be obtained by using identity (12), see deduction in Appendix B.

$$
\begin{gathered}
\Delta h=h_{2}-h_{1}=y_{N}-y_{M}=y_{\text {lev }}(S+q)-y_{\text {lev }}(q) \\
\frac{q}{c}=\operatorname{arcsinh} \frac{h_{2}-h_{1}}{2 c \cdot \sinh \frac{S}{2 c}}
\end{gathered}
$$

Substituting (13) into (11) gives the final equation (14) for the conductor curve in a modelled inclined span, expressed by the given data $\left(S, c, h_{1}\right)$ for a levelled span and $h_{2}$ obtained by $h_{2}=h_{1}+\Delta h$, where $\Delta h$ is freely chosen.

$$
y_{\text {inc }}(x)=2 c \cdot \sinh \frac{x}{2 c} \cdot \sinh \left(\frac{x-S}{2 c}+\operatorname{arcsinh} \frac{h_{2}-h_{1}}{2 c \cdot \sinh \frac{S}{2 c}}\right)+h_{1}
$$

Since $h_{1}$ is fixed, then considering (12) either $h_{2}$ or $\Delta h$ can be freely chosen for an inclined span. In other words, the span inclination is defined by the choice of either $h_{2}$ or $\Delta h$ in our case. Let us mention that $h_{1}$ and $h_{2}$ data are considered as the heights of the support points related to $x$-axis [3], but not as the tower heights.

Using (14), the sag equation concerned to an inclined span, $D_{\text {inc }}(x)$, can also be obtained as shown below:

$$
D_{\text {inc }}(x)=\frac{h_{2}-h_{1}}{S} x+h_{1}-y_{i n c}(x)
$$

$$
D_{\text {inc }}(x)=\frac{h_{2}-h_{1}}{S} x-2 c \cdot \sinh \frac{x}{2 c} \cdot \sinh \left(\frac{x-S}{2 c}+\operatorname{arcsinh} \frac{h_{2}-h_{1}}{2 c \cdot \sinh \frac{S}{2 c}}\right)
$$

Having obtained (14) and (16), the inclined span modelling is finished. Both $y_{i n c}(x)$ and $D_{i n c}(x)$ are defined on the interval $[0, S]$.

Considering the structure of (3) and (16) it can be concluded that the graph of $D_{l e v}(x)$ is a so-called inverted catenary (see in Fig. 3), while the graph of $D_{i n c}(x)$ is not, because of $\Delta h \cdot x / S$ in (16). In practice the latter graph is very similar to an inverted catenary, due to the slight difference, but mathematically it cannot be considered as that. Naturally the graphs of $y_{\text {lev }}(x)$ and $y_{\text {inc }}(x)$ are both classic catenaries.

\subsection{Deriving the difference between $D_{\text {inc }}(x)$ and $D_{\text {lev }}(x)$}

As the sag equations in levelled and inclined spans have been determined in the previous section, these can be used now to define their difference, denoted as $\Delta D(x)$. Based on (17) and considering (16) and (3), it is expressed by (18). (See deduction in Appendix C.)

$$
\Delta D(x)=D_{\text {inc }}(x)-D_{\text {lev }}(x)
$$

$$
\begin{aligned}
& \Delta D(x)=\frac{h_{2}-h_{1}}{S} x-2 c \cdot \sinh \frac{x}{2 c} . \\
& \left(\sinh \left(\frac{x-S}{2 c}+\operatorname{arcsinh} \frac{h_{2}-h_{1}}{2 c \cdot \sinh \frac{S}{2 c}}\right)+\sinh \frac{S-x}{2 c}\right)
\end{aligned}
$$

Applying identity for hyperbolic sine function given by (19) [9], then considering (20), the previous equation changes into (21).

$$
\begin{aligned}
& \sinh (x)+\sinh (y)=2 \sinh \frac{x+y}{2} \cdot \cosh \frac{x-y}{2} \cosh (-x)=\cosh (x) \\
& \Delta D(x)= \frac{h_{2}-h_{1}}{S} x-4 c \cdot \sinh \frac{x}{2 c} \\
& \cdot \sinh \left(\frac{1}{2} \operatorname{arcsinh} \frac{h_{2}-h_{1}}{2 c \cdot \sinh \frac{S}{2 c}}\right) \\
& \cdot \cosh \left(\frac{S-x}{2 c}-\frac{1}{2} \operatorname{arcsinh} \frac{h_{2}-h_{1}}{2 c \cdot \sinh \frac{S}{2 c}}\right)
\end{aligned}
$$

Using (21) it is possible to compute the difference between the catenary sags in inclined and levelled spans at any point of the span, when both the catenary constant and the span 
length are equal in two cases. The special version of (21) concerns to a mid-span, $x=S / 2$. According to (22) it is given by (23). Note that $D_{\text {lev }}(S / 2)=D_{\text {lev } \max }$, but $D_{\text {inc }}(S / 2) \neq D_{\text {inc } \max }$, and so $\triangle D(S / 2) \neq(\Delta D)_{\max }$. That is why the actual symbols are used in (22), but not $D_{\text {lev } \max }, D_{\text {inc } \max }$ and $(\Delta D)_{\max }$.

$$
\begin{aligned}
\Delta D(S / 2)= & D_{\text {inc }}(S / 2)-D_{\text {lev }}(S / 2) \\
\Delta D(S / 2)= & \frac{h_{2}-h_{1}}{2}-4 c \cdot \sinh \frac{S}{4 c} \\
& \cdot \sinh \left(\frac{1}{2} \operatorname{arcsinh} \frac{h_{2}-h_{1}}{2 c \cdot \sinh \frac{S}{2 c}}\right) \\
& \cdot \cosh \left(\frac{S}{4 c}-\frac{1}{2} \operatorname{arcsinh} \frac{h_{2}-h_{1}}{2 c \cdot \sinh \frac{S}{2 c}}\right)
\end{aligned}
$$

Let us mention that if (17) is transformed into (24) then $D_{\text {inc }}(x)$ can be obtained from the given $D_{\text {lev }}(x)$ by computing $\Delta D(x)$ with the use of (21).

$$
D_{\text {inc }}(x)=D_{\text {lev }}(x)+\Delta D(x)
$$

The previous expression can be considered as the relation between the catenary sags in inclined and levelled spans. As each part in (24) is the function of $x$, it can be applied at any point of the span.

\subsection{Practical application of the developed method}

The practical application of inclined span modelling is presented in a complex numerical task which uses the main expressions derived in sections 2.1 and 2.2. In order to bring the method closer to frequent practical cases, the sag computing is done at a mid-span in the task. With the given data $(S$, $c, h_{1}$ ) for a levelled span, the equations for the conductor curve and the sag in a levelled span have to be obtained, then also the adequate equations concerned to an inclined span with an arbitrarily selected $\Delta h$. The latter defines the span inclination. In the continuation it is necessary to determine the sag difference in a modelled inclined span and a given levelled one at a mid-span point.

\section{Task 1:}

Using the data given in Table 1 , obtain $y_{\text {lev }}(x)$ and $D_{\text {lev }}(x)$, then also $y_{\text {inc }}(x)$ and $D_{\text {inc }}(x)$ if the right-hand side support point is elevated with 200 metres (thus $h_{2}=h_{1}+200 \mathrm{~m}$ ), but $S$ and $c$ data remain unchanged. Calculate $D_{\text {lev }}(S / 2)$ and $\Delta D(S / 2)$, and then add them up to get $D_{i n c}(S / 2)$, i.e. the mid-span sag in a formed inclined span. Check the result by using (16) when $x=S / 2$. Draw the two conductor curves and their mid-span sags on the common diagram. Also draw $y_{\text {linelev }}(x)$ and $y_{\text {line inc }}(x)$, i.e. the straight lines connecting the support points in each span, to make the sag more visible.

Tab. 1. Data for levelled and inclined spans in task 1

\begin{tabular}{ccccc}
\hline Span & $S[\mathrm{~m}]$ & $h_{1}[\mathrm{~m}]$ & $h_{2}[\mathrm{~m}]$ & $c[\mathrm{~m}]$ \\
\hline Levelled & 700 & 100 & 100 & 1000 \\
Inclined & 700 & 100 & 300 & 1000 \\
\hline
\end{tabular}

\section{Solution:}

The use of (1), (3), (14) and (16) yields (25) - (28):

$$
\begin{gathered}
y_{\text {lev }}(x)=1000 \cdot \cosh \frac{x-350}{1000}-961,877819 \\
D_{\text {lev }}(x)=1061,877819-1000 \cdot \cosh \frac{x-350}{1000} \\
y_{\text {inc }}(x)=2000 \cdot \sinh \frac{x}{2000} \cdot \sinh \left(\frac{x}{2000}-0,07357\right)+100
\end{gathered}
$$

$D_{\text {inc }}(x)=0,285714 \cdot x-2000 \cdot \sinh \frac{x}{2000} \cdot \sinh \left(\frac{x}{2000}-0,07357\right)$

Inserting $x=S / 2=350 \mathrm{~m}$ into (26) and then the data from Table 1 into (23) give the following values:

$$
D_{\text {lev }}(350 \mathrm{~m})=61,878 \mathrm{~m} ; \Delta D(350 \mathrm{~m})=2,379 \mathrm{~m}
$$

The sum of the above values yields the mid-span sag in an inclined span, which has to be obtained:

$$
D_{\text {inc }}(350 \mathrm{~m})=61,878 \mathrm{~m}+2,379 \mathrm{~m}=64,257 \mathrm{~m}
$$

It is easy to check this result with the help of (28) obtained from (16). As it gives the same value, the correctness of the calculations has been improved. The results are shown in Fig. 4.

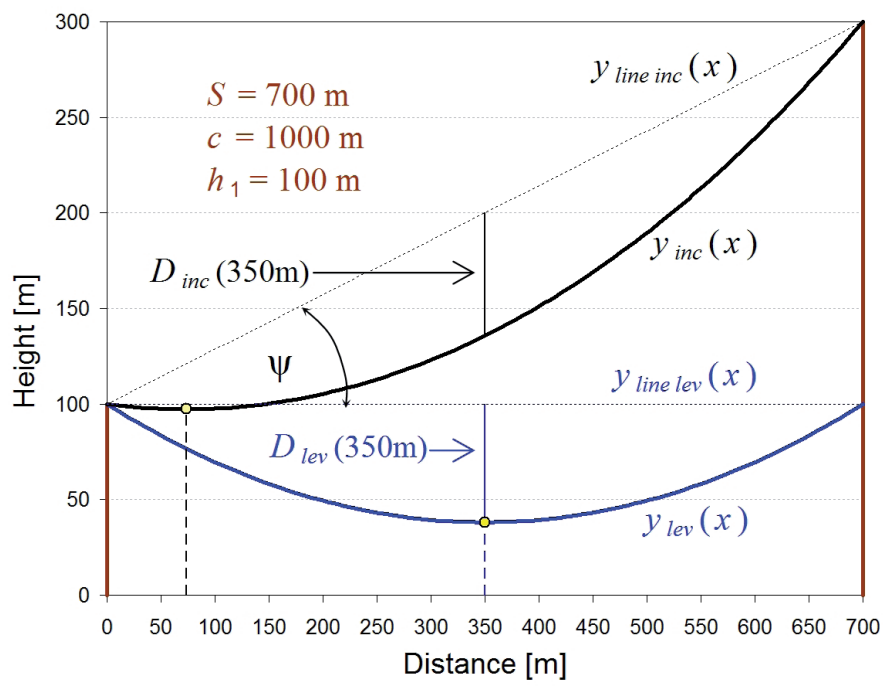

Fig. 4. Conductor curves and mid-span sags in levelled and inclined spans in task 1 


\subsection{Examination of the use of $1 / \cos \psi$ for the catenary based calculation}

In fact $1 / \cos \psi$ comes from the parabola based calculation [2, 4] and it is used for determining the parabola sag in an inclined span by a given sag in a levelled span:

$$
D_{\text {inc }}(x)=\frac{1}{\cos \psi} \cdot D_{\text {lev }}(x)
$$

The multiplier $1 / \cos \psi$ can be obtained by the following expression, where $\psi$ presents the angle of the span inclination:

$$
\frac{1}{\cos \psi}=\sqrt{1+\tan ^{2} \psi}=\sqrt{1+\left(\frac{h_{2}-h_{1}}{S}\right)^{2}}
$$

However, some earlier literatures also recommend the application of (29) when the conductor curve is considered as a catenary. Since in this case (29) is an approximate relation, its use obviously produces errors in sag calculations. The examination of it is the main goal of this section. Using the above obtained (3) and (16), the equation $E(x)$ is defined by (31).

$$
E(x)=D_{\text {inc }}(x)-\frac{1}{\cos \psi} \cdot D_{\text {lev }}(x)
$$

Thus, the value of the actual sag error can be determined at any point within the span. Applying (30) the previous equation becomes (32):

$$
E(x)=D_{i n c}(x)-\sqrt{1+\left(\frac{h_{2}-h_{1}}{S}\right)^{2}} \cdot D_{l e v}(x)
$$

In order to analyse $E(x)$, five conductor curves are drawn in Fig. 5, one in a levelled span and four others in inclined spans. As it can be seen in Table 2, the data $S, c$ and $h_{1}$ are common ones, while $h_{2}$ differs in each case. This way $E(x)$ can be analysed when the span inclination changes. The lowest points of the curves are marked as MIN $1, \ldots$, MIN 5 .

Tab. 2. Input data for curves in Fig. 5

\begin{tabular}{cccccc}
\hline & 1 & 2 & 3 & 4 & 5 \\
\hline$S[\mathrm{~m}]$ & 700 & 700 & 700 & 700 & 700 \\
$h_{1}[\mathrm{~m}]$ & 100 & 100 & 100 & 100 & 100 \\
$h_{2}[\mathrm{~m}]$ & 100 & 150 & 200 & 250 & 300 \\
$c[\mathrm{~m}]$ & 1000 & 1000 & 1000 & 1000 & 1000 \\
\hline
\end{tabular}

According to (33), the curve of $E(x)$ has been drawn in four different cases. These are denoted as $E_{1}(x), E_{2}(x), E_{3}(x), E_{4}(x)$ and shown in Fig. 6. The superscripts in (33) refer to the adequate curves and data, but note that $h_{1}{ }^{(i+1)}=h_{1}{ }^{(1)}(i=1,2,3,4)$ here.

$E_{i}(x)=D_{\text {inc }}^{(i+1)}(x)-\sqrt{1+\left(\frac{h_{2}^{(i+1)}-h_{1}^{(i+1)}}{S}\right)^{2}} \cdot D_{\text {lev }}^{(1)}(x) \quad i=1,2,3,4$

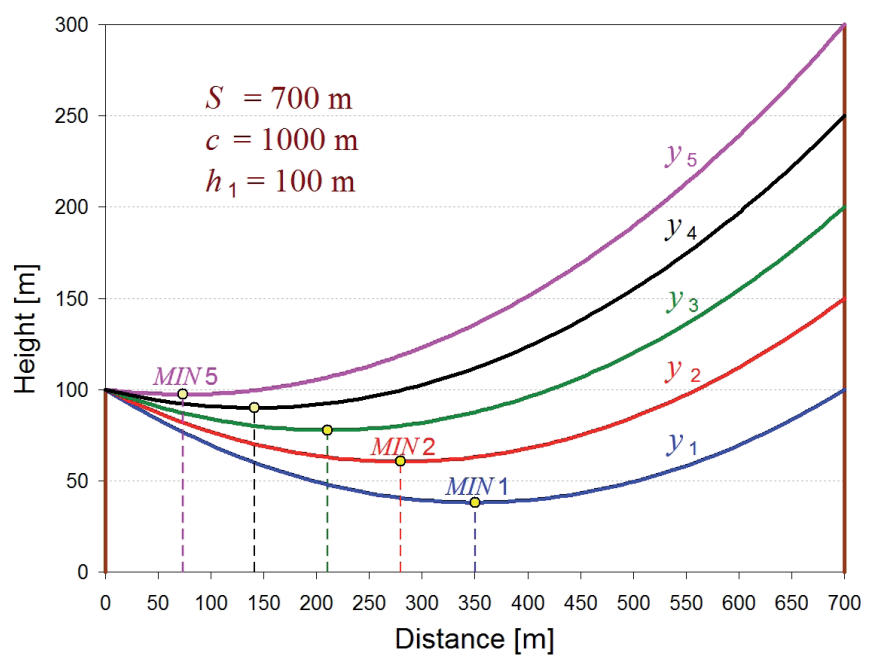

Fig. 5. One curve in a levelled span and four others in inclined spans

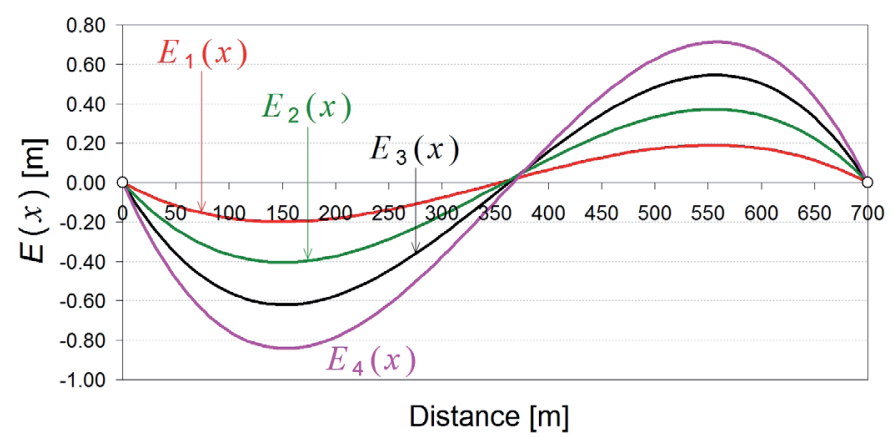

Fig. 6. $E(x)$ curves concerned to different span inclinations

Taking into consideration all curves in Fig. 6, it can be concluded that the difference between $\left|E_{\min }\right|$ and $E_{\max }$ is not significant. The locations of $E_{\min }$ and $E_{\max }$ within the span are very close to $S / 4$ and $3 S / 4$ respectively, when $h_{1}<h_{2}$. Furthermore, it is well seen that $E(x)$ has two fixed roots, $x_{1}=0$ and $x_{2}=S$, and between them also the third one. The latter slightly moves with the span inclination, toward the next nearer root, but it is always very close to a mid-span. If the mid-root is denoted as $z$, then:

$$
E(0)=E(z)=E(S)=0
$$

Since $E(x)$ changes sign within the span, $D_{\text {inc }}(x)$ is lower than $(1 / \cos \psi) D_{l e v}(x)$ in one part of the span, but in the other one it is higher. This is mathematically described in the following way:

$$
\text { If } h_{1}<h_{2} \text { then: }
$$

$$
E(x)<0 \Rightarrow D_{\text {inc }}(x)<\frac{D_{l e v}(x)}{\cos \psi} \quad \forall \quad x \in(0, z)
$$

$$
E(x)>0 \Rightarrow D_{\text {inc }}(x)>\frac{D_{\text {lev }}(x)}{\cos \psi} \quad \forall x \in(z, S)
$$


In a similar way it can be shown that the adequate relations concerned to the other type of an inclined span are given as:

$$
\begin{aligned}
& \text { If } h_{1}>h_{2} \text { then: } \\
& E(x)>0 \Rightarrow D_{\text {inc }}(x)>\frac{D_{\text {lev }}(x)}{\cos \psi} \quad \forall x \in(0, z) \\
& E(x)<0 \Rightarrow D_{\text {inc }}(x)<\frac{D_{\text {lev }}(x)}{\cos \psi} \quad \forall x \in(z, S)
\end{aligned}
$$

Thus, signs of the sag errors on the intervals $(0, z)$ and $(z, S)$ depend on the type of the inclined span, $h_{1}<h_{2}$ or $h_{1}>h_{2}$. It is worth mentioning that errors in sag calculation directly produce errors in clearance calculation. Hence, the use of the approximate relation given by (29) is unfavourable from different aspects. That is why the application of an exact equation for computing $D_{i n c}(x)$ given by (16) is recommended instead of (29).

\subsection{Analysis of the expression for the maximum sag of the catenary}

Taking into consideration the above mentioned, now expression (39) can be analysed, which occurs in some earlier literatures, though its deduction has never been shown and explained.

$$
b=\frac{c}{a} \cdot \frac{2 \sigma}{\gamma} \sinh ^{2} \frac{a \gamma}{4 \sigma}
$$

Here $b$ is a maximum sag in anclined span, $c$ presents a length of the straight line connecting the support points, $a$ is a span length and finally the quotient $\sigma / \gamma$ defines a catenary constant. Expressing $c$ by the Pythagorean theorem and using labels from the recent paper, (39) can be rewritten as:

$$
D_{\text {inc } \max }=\frac{\sqrt{S^{2}+\left(h_{2}-h_{1}\right)^{2}}}{S} \cdot 2 c \cdot \sinh ^{2} \frac{S}{4 c}
$$

The quotient in the previous formula is in fact $1 / \cos \psi$ multiplier from (29), while the remaining part of the formula presents the maximum sag of the catenary in a levelled span [3], due to (41).

$$
D_{\text {lev } \max }=c \cdot\left(\cosh \frac{S}{2 c}-1\right)=2 c \cdot \sinh ^{2} \frac{S}{4 c}
$$

Thus, (39) can be expressed in the following form:

$$
D_{\text {inc } \max }=\frac{1}{\cos \psi} \cdot D_{\text {lev } \max }
$$

This formula can be considered only as an approximate one, since it contains evident mathematical inexactnesses. Taking into account the above discussed, the use of $1 / \cos \psi$ for determining the catenary sag in an inclined span from the given sag in a levelled one does not give exact results. Moreover, since (42) is in fact (29) concerned to a mid-span, $D_{\text {inc }}(S / 2)$ should stay in (42) instead of $D_{\text {inc max }}$. It is because the maximum sag of the catenary in an inclined span is slightly moved from the mid-span, while in a levelled span it is not. Therefore, (39) cannot be an exact relation at all. The errors caused using (39) are not significant in spans with low inclination, but in steep spans they can be. However, the application of an exact equation given by (43) [3] is recommended instead of (39) or (42).

$$
\begin{aligned}
& D_{\text {inc max }}=2 c \cdot\left(\frac{h_{2}-h_{1}}{2 S}\left(\frac{S}{2 c}-\operatorname{arcsinh} \frac{h_{2}-h_{1}}{2 c \cdot \sinh \frac{S}{2 c}}+\operatorname{arcsinh} \frac{h_{2}-h_{1}}{S}\right)\right. \\
& \left.-\sinh ^{2}\left(\frac{1}{2} \operatorname{arcsinh} \frac{h_{2}-h_{1}}{S}\right)+\sinh ^{2}\left(\frac{1}{2}\left(\frac{S}{2 c}-\operatorname{arcsinh} \frac{h_{2}-h_{1}}{2 c \cdot \sinh \frac{S}{2 c}}\right)\right)\right)
\end{aligned}
$$

The previous formula concerns to inclined spans, i.e. when $h_{1} \neq h_{2}$. If $h_{1}=h_{2}$, the span is then levelled and thus (43) changes into (41).

\subsection{Relation between $D_{\text {inc } \max }$ and $D_{\text {lev max }}$}

Returning to (42), in section 2.5 it is considered as an approximate formula for computing the maximum sag of the catenary in inclined spans and then the adequate exact formula has been given. Note that taking into account its structure, (42) can also be considered as an approximate relation between the maximum sags of the catenary in inclined and levelled spans. Since in this case the adequate exact relation is presently not available in literatures, it is worth determining it here using the above explained method for modelling an inclined span. This process needs three steps:

- Deriving $D_{i n c}(x)$ so that it contains $D_{l e v \max }$ in the final equation,

- Finding the location of $D_{i n c \max }$, i.e. $x_{C}$, solving the equation $\left(D_{\text {inc }}(x)\right)^{\prime}=0$,

- Inserting $x_{C}$ into $D_{i n c}(x)$ to get $D_{\text {inc } \max }$.

Here the use of (2) is recommended, because it contains $D_{\text {lev } \max }$. Expression (44) is derived in order to simplify the determination of the first derivative of $D_{\text {inc }}(x)$. Thus, $\left(D_{\text {inc }}(x)\right)^{\prime}$ is given by (45). (A complete deduction for all three steps is given in Appendix D.)

$$
\begin{aligned}
D_{\text {inc }}(x) & =\frac{h_{2}-h_{1}}{S} x-c \cdot \cosh \frac{x-S / 2+q}{c} \\
& +c \cdot \cosh \frac{q-S / 2}{c}-c \cdot \cosh \frac{S}{2 c}+c+D_{\text {lev } \max }
\end{aligned}
$$

$$
\frac{d}{d x} D_{i n c}(x)=\frac{h_{2}-h_{1}}{S}-\sinh \frac{x-S / 2+q}{c}
$$

Solving (46) yields $x_{C}$ :

$$
\frac{d}{d x} D_{i n c}(x)=0 \quad \Rightarrow \quad x_{C}
$$




$$
x_{C}=\frac{S}{2}-c \cdot \operatorname{arcsinh} \frac{h_{2}-h_{1}}{2 c \cdot \sinh \frac{S}{2 c}}+c \cdot \operatorname{arcsinh} \frac{h_{2}-h_{1}}{S}
$$

The use of (47) in (44) gives the final equation (48) which presents the exact relation between the maximum sags of the catenary in inclined and levelled spans. Here $x_{M I N}$ is applied to simplify the expression. It presents the $x$-coordinate of the vertex point of the catenary in an inclined span and is given by an available formula (49) [3]. Notice that $x_{M I N}=x_{M I N}(S, c, \Delta h)$, and thus $D_{\text {inc } \max }=D_{\text {inc } \max }\left(D_{\text {lev } \max }, S, c, \Delta h\right)$.

$$
\begin{aligned}
D_{\text {inc } \max }= & D_{\text {lev } \max } \\
& +\frac{h_{2}-h_{1}}{S} \cdot\left(x_{M I N}+c \cdot \operatorname{arcsinh} \frac{h_{2}-h_{1}}{S}\right) \\
& -2 c \cdot \sinh ^{2} \frac{S}{4 c} \\
& +2 c \cdot \sinh \left(\frac{1}{2 c}\left(x_{M I N}+c \cdot \operatorname{arcsinh} \frac{h_{2}-h_{1}}{S}\right)\right) \\
& \cdot \sinh \left(\frac{1}{2 c}\left(x_{M I N}-c \cdot \operatorname{arcsinh} \frac{h_{2}-h_{1}}{S}\right)\right) \\
& x_{\text {MIN }}=\frac{S}{2}-c \cdot \operatorname{arcsinh} \frac{h_{2}-h_{1}}{2 c \cdot \sinh \frac{S}{2 c}}
\end{aligned}
$$

The simplified form of (48) is given by (50) where $\Delta D_{\text {max }}=\Delta D_{\text {max }}(S, c, \Delta h)$ presents the difference between the maximum sags of the catenary in inclined and levelled spans. If $D_{\text {lev max }}$ is given, then $D_{\text {inc max }}$ can be obtained by computing $\Delta D_{\max }$ and adding it up to $D_{\text {lev } \max }$.

$$
D_{\text {inc } \max }=D_{\text {lev } \max }+\Delta D_{\text {max }}
$$

The applicability of (48) will be presented regarding to five catenaries drawn in Fig. 5 and using the input data from Table 2 (section 2.4). Applying (41) and (48), the maximum sags for all catenaries drawn in Fig. 5 are computed and then listed in Table 3:

Tab. 3. Maximum sags of the catenaries drawn in Fig. 5

\begin{tabular}{cccc}
\hline Curve & $\begin{array}{c}D_{\text {lev } \max } \\
{[\mathrm{m}]}\end{array}$ & $\begin{array}{c}D_{\text {inc } \max } \\
{[\mathrm{m}]}\end{array}$ & $\begin{array}{c}D_{\text {inc } \max }-D_{\text {lev } \max } \\
{[\mathrm{m}]}\end{array}$ \\
\hline$y_{1}$ & 61,87782 & - & - \\
$y_{2}$ & - & 62,03023 & 0,15241 \\
$y_{3}$ & - & 62,48522 & 0,60740 \\
$y_{4}$ & - & 63,23626 & 1,35844 \\
$y_{5}$ & - & 64,27297 & 2,39515 \\
\hline
\end{tabular}

It is evident that the maximum sag of the catenary increases with the span inclination. Let us mention that each of five sags has different location within the span, even though $S$ and $c$ are common data. This is an important difference in comparison to a parabola, since its maximum sag is always located at a mid-span independently of the span inclination or the height difference of the support points.

\section{Future work}

A logical continuation of the recent paper is the application of the developed method for a parabola. All the necessary input data are the same as in the catenary case, except for the catenary constant. Instead of that the maximum sag of the parabola has to be used. Naturally, much simpler final equations are expected since the parabola is an algebraic function, while the catenary is a transcendental one.

\section{Conclusions}

This paper shows in details how to model an inclined span by the given basic data of a levelled one, when the span length and the catenary constant are common data. The equations for the conductor curve and the sag are given in both levelled and inclined spans. Also an exact formula for computing the maximum sag of the catenary has been shown. Besides, the corresponding approximate formula from earlier literatures has been analysed and appropriately discussed. Furthermore, some special features of the catenary are introduced. Based on chapter 2 , it is evident that the function given as a quotient of $D_{\text {inc }}(x)$ and $D_{\text {lev }}(x)$ in the interval $(0, S)$ is not a constant in the case of the catenary. It is expressed by (51):

$$
\frac{D_{\text {inc }}^{\text {(cat) }}(x)}{D_{\text {lev }}^{\text {(cat) }}(x)} \neq \text { const. } \quad 0<x<S
$$

Taking into consideration the adequate relation concerned to a parabola given by (52) [2], a remarkable difference between the parabola and the catenary based calculations has been explored.

$$
\frac{D_{\text {inc }}^{(\mathrm{par})}(x)}{D_{\text {lev }}^{\text {(par) }}(x)}=\text { const. } \quad 0<x<S
$$

The following results are worth to be emphasized among the other ones provided in this paper:

- Equation for computing the difference between the catenary sags in inclined and levelled spans at any point of the span,

- Relation between the maximum sags of the catenary in inclined and levelled spans.

These are mathematically exact unique expressions related to a catenary, which are important in the field of OHL design. 


\section{References}

1 Grigsby L. L., Electric Power Generation, Transmission and Distribution, Taylor \& Fransis Group LLC., (2012).

2 Hatibovic A., Derivation and Analysis of the Relation between Conductor Sags in Inclined and Levelled Spans Based on Known Data of the Latter, CIGRÉ $45^{\text {th }}$ International Conference on Large High Voltage Electric Systems, (2014).

3 Hatibovic A., Derivation of Equations for Conductor and Sag Curves of an Overhead Line Based on a Given Catenary Constant, Periodica Polytechnica Electrical Engineering and Computer Science, 58 (1), pp. 23-27, (2014). DOI: $10.3311 /$ PPee.6993

4 Hatibovic A., Integral Calculus Usage for Conductor Length Determination on the Basis of Known Maximal Sag of a Parabola, Periodica Polytechnica Electrical Engineering and Computer Science, 56 (2), pp. 35-41, (2012).

DOI: $\underline{10.3311 / \text { PPee. } 7076}$
$5 \quad$ Kiessling F., Nefzger P., Nolasco J. F., Kaintzyk U., Overhead Power Lines, Planning, Design, Construction, Springer, 2003.

6 Mahajan S. M., Singareddy U. M., A Real-Time Conductor Sag Measurement System Using a Differential GPS, IEEE Transactions on Power Delivery, 27 (2), pp. 475-480, (2012).

DOI: $10.1109 /$ TPWRD. 2011.2181963

7 Malhara S., Vittal V., Mechanical State Estimation of Overhead Transmission Lines Using Tilt Sensors, IEEE Transactions on Power Systems, 25 (3), pp. 1282-1290, (2010). DOI: $\underline{10.1109 / T P W R S ~ .2009 .2038703}$

8 Sakala J. D., Improved Calculation of Sag for a Conductor Supported at Unequal Heights, International Journal of Electrical Engineering Education, 45 (4), pp. 327-335, (2008).

9 http://perso.univ-rennes1.fr/maximilian.bauer/AN1/fonctions hyperboliques.pdf

10 http://www.oregon.gov/Energy/Siting/docs/Meeting_Materials/031513/ItemG_Idaho_Power_presentation_031513.pdf

\section{Appendix A}

$$
\begin{gathered}
y_{\text {inc }}(x)=(A Q)_{\text {curve }}=y_{\text {lev }}(x+q)+h_{1}-y_{\text {lev }}(q) \\
y_{\text {lev }}(x+q)=c \cdot \cosh \frac{x+q-S / 2}{c}-c \cdot \cosh \frac{S}{2 c}+h_{1} \\
y_{\text {lev }}(q)=c \cdot \cosh \frac{q-S / 2}{c}-c \cdot \cosh \frac{S}{2 c}+h_{1} \\
y_{\text {inc }}(x)=c \cdot\left(\cosh \frac{x+q-S / 2}{c}-\cosh \frac{q-S / 2}{c}\right)+h_{1} \\
\cosh (x)-\cosh (y)=2 \sinh \frac{x+y}{2} \cdot \sinh \frac{x-y}{2} \\
y_{\text {inc }}(x)=2 c \cdot \sinh \frac{x}{2 c} \cdot \sinh \left(\frac{x-S}{2 c}+\frac{q}{c}\right)+h_{1}
\end{gathered}
$$

\section{Appendix B}

$$
\begin{gathered}
h_{2}-h_{1}=y_{N}-y_{M}=y_{\text {lev }}(S+q)-y_{\text {lev }}(q) \\
y_{N}=c \cdot \cosh \frac{S / 2+q}{c}-c \cdot \cosh \frac{S}{2 c}+h_{1} \\
y_{M}=c \cdot \cosh \frac{q-S / 2}{c}-c \cdot \cosh \frac{S}{2 c}+h_{1} \\
h_{2}-h_{1}=c \cdot\left(\cosh \frac{q+S / 2}{c}-\cosh \frac{q-S / 2}{c}\right)
\end{gathered}
$$




$$
\begin{gathered}
\cosh (x)-\cosh (y)=2 \sinh \frac{x+y}{2} \cdot \sinh \frac{x-y}{2} \\
h_{2}-h_{1}=2 c \cdot \sinh \frac{q}{c} \cdot \sinh \frac{S}{2 c} \\
\sinh \frac{q}{c}=\frac{h_{2}-h_{1}}{2 c \cdot \sinh \frac{S}{2 c}} \\
\operatorname{arcsinh}\left(\sinh \frac{q}{c}\right)=\operatorname{arcsinh} \frac{h_{2}-h_{1}}{2 c \cdot \sinh \frac{S}{2 c}} \\
\frac{q}{c}=\operatorname{arcsinh} \frac{h_{2}-h_{1}}{2 c \cdot \sinh \frac{S}{2 c}}
\end{gathered}
$$

\section{Appendix C}

$$
\begin{gathered}
D_{\text {lev }}(x)=c \cdot\left(\cosh \frac{S}{2 c}-\cosh \frac{x-S / 2}{c}\right) \\
\cosh (x)-\cosh (y)=2 \sinh \frac{x+y}{2} \cdot \sinh \frac{x-y}{2} \\
D_{l e v}(x)=2 c \cdot \sinh \frac{x}{2 c} \cdot \sinh \frac{S-x}{2 c} \\
D_{\text {inc }}(x)=\frac{h_{2}-h_{1}}{S} x-2 c \cdot \sinh \frac{x}{2 c} \cdot \sinh \left(\frac{x-S}{2 c}+\operatorname{arcsinh} \frac{h_{2}-h_{1}}{2 c \cdot \sinh \frac{S}{2 c}}\right) \\
\Delta D(x)=D_{\text {inc }}(x)-D_{\text {lev }}(x) \\
\Delta D(x)=\frac{h_{2}-h_{1}}{S} x-2 c \cdot \sinh \frac{x}{2 c} \cdot\left(\begin{array}{c}
\left.\sinh \left(\frac{x-S}{2 c}+\operatorname{arcsinh} \frac{h_{2}-h_{1}}{2 c \cdot \sinh \frac{S}{2 c}}\right)+\sinh \frac{S-x}{2 c}\right)
\end{array}\right)
\end{gathered}
$$

\section{Appendix D}

Step 1

$$
\begin{gathered}
D_{i n c}(x)=\frac{h_{2}-h_{1}}{S} x+h_{1}-y_{i n c}(x) \\
D_{i n c}(x)=\frac{h_{2}-h_{1}}{S} x+h_{1}-\left(y_{l e v}(x+q)+h_{1}-y_{l e v}(q)\right)
\end{gathered}
$$




$$
\begin{gathered}
y_{l e v}(x+q)=c \cdot \cosh \frac{x-S / 2+q}{c}-c+h_{1}-D_{\text {lev } \max } \\
y_{\text {lev }}(q)=c \cdot \cosh \frac{q-S / 2}{c}-c \cdot \cosh \frac{S}{2 c}+h_{1} \\
D_{\text {inc }}(x)=\frac{h_{2}-h_{1}}{S} x-c \cdot \cosh \frac{x-S / 2+q}{c}+c \cdot \cosh \frac{q-S / 2}{c}-c \cdot \cosh \frac{S}{2 c}+c+D_{\text {lev max }} \\
D_{\text {inc }}(x)=D_{\text {lev } \max }+\frac{h_{2}-h_{1}}{S} x-2 c \cdot \sinh ^{2} \frac{S}{4 c}+c \cdot \cosh \frac{q-S / 2}{c}-c \cdot \cosh \frac{x-S / 2+q}{c}
\end{gathered}
$$

\section{Step 2}

$$
\begin{gathered}
\frac{d}{d x} D_{\text {inc }}(x)=\frac{h_{2}-h_{1}}{S}-\sinh \frac{x-S / 2+q}{c} \\
\frac{d}{d x} D_{\text {inc }}(x)=0 \Rightarrow x_{C} \\
\frac{h_{2}-h_{1}}{S}-\sinh \frac{x_{C}-S / 2+q}{c}=0 \\
\sinh \frac{x_{C}-S / 2+q}{c}=\frac{h_{2}-h_{1}}{S} \\
\frac{x_{C}-S / 2+q}{c}=\operatorname{arcsinh} \frac{h_{2}-h_{1}}{S} \\
x_{C}=\frac{\sinh _{C}-S / 2+q}{c}-c \cdot \operatorname{arcsinh} \frac{x_{2}-h_{1}}{2 c \cdot \sinh \frac{S}{2 c}}+c \cdot \operatorname{arcsinh} \frac{h_{2}-h_{1}}{S} \\
\frac{S}{2}-q+c \cdot \operatorname{arcsinh} \frac{h_{2}-h_{1}}{S} \\
q=c \cdot \operatorname{arcsinh} \frac{h_{2}-h_{1}}{2 c \cdot \sinh \frac{S}{2 c}}
\end{gathered}
$$

Step 3

$$
\begin{gathered}
D_{\text {inc } \max }=D_{\text {inc }}\left(x_{C}\right) \\
D_{\text {inc max }}=D_{\text {lev max }}+\frac{h_{2}-h_{1}}{S} x_{C}-2 c \cdot \sinh ^{2} \frac{S}{4 c}+c \cdot\left(\cosh \frac{q-S / 2}{c}-\cosh \frac{x_{C}-S / 2+q}{c}\right)
\end{gathered}
$$




$$
\begin{aligned}
& \cosh (x)-\cosh (y)=2 \sinh \frac{x+y}{2} \cdot \sinh \frac{x-y}{2} \\
& c \cdot\left(\cosh \frac{q-S / 2}{c}-\cosh \frac{x_{C}-S / 2+q}{c}\right)=2 c \cdot \sinh \frac{x_{C}-S+2 q}{2 c} \cdot \sinh \frac{-x_{C}}{2 c} \\
& \sinh (-x)=-\sinh (x) \\
& c \cdot\left(\cosh \frac{q-S / 2}{c}-\cosh \frac{x_{C}-S / 2+q}{c}\right)=2 c \cdot \sinh \frac{x_{C}}{2 c} \cdot \sinh \frac{S-x_{C}-2 q}{2 c} \\
& D_{\text {inc } \max }=D_{\text {lev } \max }+\frac{h_{2}-h_{1}}{S} \cdot\left(\frac{S}{2}-c \cdot \operatorname{arcsinh} \frac{h_{2}-h_{1}}{2 c \cdot \sinh \frac{S}{2 c}}+c \cdot \operatorname{arcsinh} \frac{h_{2}-h_{1}}{S}\right)-2 c \cdot \sinh ^{2} \frac{S}{4 c}+ \\
& +2 c \cdot \sinh \left(\frac{1}{2 c}\left(\frac{S}{2}-c \cdot \operatorname{arcsinh} \frac{h_{2}-h_{1}}{2 c \cdot \sinh \frac{S}{2 c}}+c \cdot \operatorname{arcsinh} \frac{h_{2}-h_{1}}{S}\right)\right) . \\
& \cdot \sinh \left(\frac{1}{2 c}\left(S-\left(\frac{S}{2}-c \cdot \operatorname{arcsinh} \frac{h_{2}-h_{1}}{2 c \cdot \sinh \frac{S}{2 c}}+c \cdot \operatorname{arcsinh} \frac{h_{2}-h_{1}}{S}\right)-2 c \cdot \operatorname{arcsinh} \frac{h_{2}-h_{1}}{2 c \cdot \sinh \frac{S}{2 c}}\right)\right) \\
& D_{\text {inc } \max }=D_{\text {lev } \max }+\frac{h_{2}-h_{1}}{S} \cdot\left(\frac{S}{2}-c \cdot \operatorname{arcsinh} \frac{h_{2}-h_{1}}{2 c \cdot \sinh \frac{S}{2 c}}+c \cdot \operatorname{arcsinh} \frac{h_{2}-h_{1}}{S}\right)-2 c \cdot \sinh ^{2} \frac{S}{4 c}+ \\
& +2 c \cdot \sinh \left(\frac{1}{2 c}\left(\frac{S}{2}-c \cdot \operatorname{arcsinh} \frac{h_{2}-h_{1}}{2 c \cdot \sinh \frac{S}{2 c}}+c \cdot \operatorname{arcsinh} \frac{h_{2}-h_{1}}{S}\right)\right) \text {. } \\
& \cdot \sinh \left(\frac{1}{2 c}\left(\frac{S}{2}-c \cdot \operatorname{arcsinh} \frac{h_{2}-h_{1}}{2 c \cdot \sinh \frac{S}{2 c}}-c \cdot \operatorname{arcsinh} \frac{h_{2}-h_{1}}{S}\right)\right) \\
& x_{M I N}=\frac{S}{2}-c \cdot \operatorname{arcsinh} \frac{h_{2}-h_{1}}{2 c \cdot \sinh \frac{S}{2 c}} \\
& D_{\text {inc } \max }=D_{\text {lev } \max }+\frac{h_{2}-h_{1}}{S} \cdot\left(x_{\text {MIN }}+c \cdot \operatorname{arcsinh} \frac{h_{2}-h_{1}}{S}\right)-2 c \cdot \sinh ^{2} \frac{S}{4 c}+ \\
& +2 c \cdot \sinh \left(\frac{1}{2 c}\left(x_{M I N}+c \cdot \operatorname{arcsinh} \frac{h_{2}-h_{1}}{S}\right)\right) \cdot \sinh \left(\frac{1}{2 c}\left(x_{M I N}-c \cdot \operatorname{arcsinh} \frac{h_{2}-h_{1}}{S}\right)\right) \\
& D_{i n c \max }=D_{\text {lev } \max }+\Delta D_{\text {max }}
\end{aligned}
$$

